\title{
Urinary Calculi and Ulcerative Colitis
}

\author{
R. C. BENNETT, E. S. R. HUGHES
}

\section{Summary}

A survey of 458 patients treated for ulcerative colitis either medically or surgically by colectomy and the establishment of an ileostomy has provided further evidence that in Australia at least there is a significant association between ulcerative colitis and urinary calculi. The actual risk to the unoperated patient is not particularly high but is in the vicinity of $4 \%$. The survey further confirms that the risk continues after colectomy and ileostomy, when the overall incidence of stones is roughly doubled. Finally, suggestive though admittedly somewhat inconclusive evidence supports the proposition that uric acid stones are more common in this group of patients.

\section{Introduction}

In addition to the many well recognized complications, ulcerative colitis may be associated with one or more of many local and systemic conditions, the incidence of which ranges upwards from about $1 \%$ (Goligher, de Dombal, Watts, and Watkinson, 1968). In a previous report (Bennett and Jepson, 1966) it was suggested that urinary calculi, particularly those composed of uric acid, formed another less well recognized association of this disease. In particular, the evidence suggested that unlike most other such conditions the risk of calculi actually increased after the establishment of an ileostomy. Evidence of this association had also been produced in North America (Deren, Porush, Levitt, and Khilnani, 1962) and Europe (Badenoch, 1960; Maratka and Nedbal, 1964). More recently, other well documented reports of large series of patients suffering from ulcerative colitis have failed to show any connexion between this disease and urinary calculi (Edwards and Truelove, 1964; Goligher et al., 1968). Furthermore, Daly (1968) did not find any urinary stones in his series of patients reviewed after proctocolectomy. To further complicate the picture Gelzayd, Breuer, and Kirsner (1968), in North America, found stones in $18 \%$ of patients with ileostomies, and Ritchie (1971) found an incidence of $4.3 \%$ in patients treated by excisional surgery in the London area.

Because of the conflicting nature of these reports and the fact that our earlier observations were based on a relatively small series of 72 patients - all with ileostomies-and the possibility of geographical, climatic, or other factors playing a part, a further, more extensive survey was undertaken in the State of Victoria. The findings provide additional support for the propositionat least within the Southern States of Australia.

\section{Patients and Method}

A postal survey was conducted on 558 patients suffering from ulcerative colitis and resident in the State of Victoria. All patients had evidence of involvement to a varying degree of the \footnotetext{
University of Melbourne, St. Vincent's Hospital, Melbourne,
Australia

R. C. BENNETT, M.s., F.R.c.s., Professor of Surgery

Royal Melbourne Hospital, Melbourne, Australia

E. S. R. HUGHES, M.S., F.R.c.s., Surgeon
}

proximal colon in addition to the rectum and could be divided into two groups according to their previous treatment. Altogether 148 patients had been managed conservatively by one of us (E.S.R.H.), and the remaining 410 patients were members of the Victorian branch of the Ileostomy Association and had had a colectomy and ileostomy performed by a number of different surgeons at varying intervals of time before the survey.

All patients were contacted by letter and asked a series of questions relating to the duration of symptoms, the time of operation (if any), whether or not they had ever passed a urinary stone, and if so what relation this bore either to the onset of their colonic symptoms or to their operation or to both. Whenever a reply suggested that a urinary calculus might have been passed verification was sought by further contact with the patient, the surgeon, the general practitioner, or any other doctor who may have been concerned in the patient's management at the time. Whenever possible an attempt was made to define the nature of the calculus by its radiographic appearances or biochemical composition or both.

To provide at least some measure of the incidence of urinary calculi in the population at large the medical records of inpatients at St. Vincent's Hospital for the period 1965-7 inclusive were reviewed and the proportion of uric acid calculi in the overall stone population was determined.

\section{Results}

\section{PATIENTS TREATED MEDICALLY}

Of the 148 patients contacted by letter $125(84 \%)$ replied. From 12 positive replies confirmation of the occurrence of calculi was obtained in eight patients $(6.4 \%)$. The time at which the calculi were passed in relation to the onset of the colitic symptoms was ascertained in each case. In three patients the passage of stones preceded the onset of colitis, but in the remaining five $(4 \%$ of the group under investigation) the calculi occurred for the first time after the onset of symptoms. Chemical analysis of the stones was undertaken in three cases, and in each instance the stone was found to consist of a mixture containing urates, calcium, and oxalate.

\section{PATIENTS WITH ILEOSTOMIES}

Of the 410 patients contacted $352(86 \%)$ replied. It was found that 19 of these patients had ileal bladders and that at no stage had they suffered from ulcerative colitis. They were subsequently excluded from the study, thus leaving 333 patients with ileostomies after colectomy for ulcerative colitis. From 38 positive replies confirmation of urinary calculi was obtained in 33 patients $(10 \%)$. Detailed analysis of these patients showed that in two instances the stones were passed before the onset of colitis, and in a further three patients other aetiological factors were thought to be important (two with bladder-neck obstruction and one with gout). The remaining 28 patients $(8.4 \%)$ had passed calculi after the onset of colitis and in the absence of other known aetiological agents.

Further consideration of the 33 patients with calculi and the time at which the stones were passed showed that while calculi had occurred before the onset of colitic symptoms in only two instances they presented after the onset of colitis but before the establishment of an ileostomy in 13 patients $(4 \%)$. Four of these patients suffered further episodes of stone formation after the 
establishment of an ileostomy. It was also found that stones had occurred after the establishment of an ileostomy in a total of 22 patients $(7 \%)$. These 333 patients virtually act as their own controls. The figures clearly indicate a steadily increasing incidence of stone formation with the development of the disease and subsequent colectomy with the creation of an ileostomy. Multiple recurrent calculi occurred in 12 of the 28 patients.

Unfortunately chemical analysis was performed on a disappointingly small proportion of the calculi. Four were certainly composed of calcium, and an additional three were seen to be opaque on $x$-ray examination and were therefore probably also composed of calcium salts. Two calculi were found on analysis to consist of uric acid (though one of these patients also suffered from gout). An additional two calculi were found on $x$-ray examination to be non-opaque and therefore might also have been largely composed of uric acid or urates. The numbers are obviously too small to draw dogmatic conclusions but it is likely that four of the 11 calculi under consideration were uric acid in nature.

During the years 1965-7 31,994 patients were admitted to St. Vincent's Hospital, and of these $223(0 \cdot 7 \%)$ had urinary calculi as either a primary or a secondary diagnosis. With regard to chemical analysis of the stones only five out of 80 so investigated during this time proved to be uric acid in nature-a relative incidence of $6.5 \%$ among the overall stone population.

\section{Discussion}

The size of this survey and the inclusion of patients who were treated medically as well as those operated on should provide a more reliable index to the risk of calculus formation than the earlier reivew of 72 patients with ileostomies (Bennett and Jepson, 1966). Furthermore, the survey covered a different community and patients operated on by a large number of different surgeons. Though the investigation consisted of a postal survey a very good response rate was achieved when considering the large number of patients circularized, and it is considered that replies from $85 \%$ of the overall group under investigation provides a satisfactory basis for assessment. Moreover, while the initial approach to the patient was by means of a letter all possible positive replies were doubly checked with both the patient and the attending doctors, so that only patients in whom the occurrence of stones could be accurately verified were included in the positive group.

Out of all 458 patients included in both parts of the survey only five had urinary stones before the onset of colitis. Moreover, in the two groups of patients treated medically either $(a)$ as the only form of therapy or $(b)$ as a preliminary to subsequent surgery there was a significant incidence of urinary calculus formation after the onset of colitic symptoms-about $4 \%$, a figure which was found in both groups. Furthermore, the 333 patients with ileostomies showed an incidence of calculus formation which increased first after the onset of colitis and again after the operation. While only two patients from this group definitely had stones before the onset of colitis 31 patients ultimately suffered from calculus formation-either subsequent to the onset of the disease alone (13) or after surgery (22) or both. In only three patients could other aetiological factors be invoked. The final incidence of $8.4 \%$ in patients with ileostomies but without other causes is roughly double that found in patients treated by medical means alone.

Lastly, it is important to note the high incidence of multiple calculi which occurred in almost half of the patients in this group. This is another point of pronounced similarity with the previous series (Bennett and Jepson, 1966) and appears to be particularly common with uric acid stones. Furthermore, it certainly suggests some underlying aetiological factor, such as the fluid and electrolyte loss in the diarrhoea of unoperated patients and in the ileostomy fluid after operation. Lavan, Neale, and Posen
(1971) also noted the frequency of diarrhoea among patients with both calcium and uric acid stones.

Previous reports of urinary calculi in patients treated for ulcerative colitis with or without surgery suggested a relatively high incidence of uric acid stones (Deren et al., 1962; Maratka and Nedbal, 1964; Bennett and Jepson, 1966). Unfortunately in this series we were not able to determine the composition of the stones in the vast majority of patients. It seems reasonably certain, however, that four of the 11 patients in whom some evidence was available had uric acid calculi. Even allowing for the one patient with associated gout this is a greater proportion of uric acid calculi than one might normally expect in this community. The relative incidence of uric acid calculi at St. Vincent's Hospital during the three-year period under review was $6.5 \%$, which is comparable to the figure of $6 \%$ quoted by Proudman (1964) for the relative incidence of uric acid calculi in Adelaide, and that of $4.7 \%$ in Sydney (Lavan et al., 1971). Probably the highest naturally occurring relative incidence of uric acid stones (33\%) was reported by Atsmon, De Vries, and Frank (1963) from Israel.

It is not easy to obtain an accurate estimate of the incidence of urinary calculi in the population at large. The figures available from St. Vincent's Hospital can be accepted only as a guide in this respect, for they deal with a selected portion of the population-namely, those admitted to hospital-and also relate specifically to a certain period of time. Williams (1966) quoted a figure of about $1 \%$ for the normal population in Great Britain. If we use our own patients as controls only five of $\mathbf{4 5 8}$ patients had calculi before the onset of colitis-an incidence of $1 \cdot 1 \%$. When any of these criteria are used as a guide it is clear that patients with ulcerative colitis run a somewhat higher risk of calculus formation (about $4 \%$ in both parts of this study), and in patients undergoing colectomy and ileostomy formation this risk is ultimately doubled-about $8 \%$. It is natural to assume that the risk to the individual patient will be greater the longer the period of symptoms and the longer the time since the construction of the ileostomy.

Review of the literature now shows obvious differences of opinion regarding the incidence of urinary stone formation in this disease. It is hard to satisfactorily explain these discrepancies. Our local experience, however, has shown quite clearly that unless the patient is specifically asked about the possibility of urinary stones he is unlikely to mention this at any follow-up review relating to his ileostomy or ulcerative colitis. The patient has usually not associated the two conditions, and indeed more often than not was treated by different doctors on each occasion. Frequently we found that the surgeon or consultant advising the patient with regard to the colitis was unaware of the secondary problem of urinary stones.

With regard to the investigation by Daly (1968) evidence of calculus formation was sought in patients with ileostomies by taking plain $x$-ray films of the abdomen at the time of review which, of course, would show only opaque calculi, thus overlooking the possible presence of radiolucent uric acid stones. Furthermore, no mention was made of any attention being paid to the past history in the assessment of this complication. If this is so then it may provide further reason for the discrepancy between his findings and those in this series. The reviews undertaken by Edwards and Truelove (1964) and by Goligher et al. (1968) are extremely well documented studies, and in neither was a significant incidence of renal disease or renal calculi noted; however, it is again difficult to assess with what diligence this association was sought. Though the accuracy of their findings is not disputed one cannot discount the reports from North America (Deren et al., 1962), Europe (Maratka and Nedbal, 1964), and Australia. In considering a possible reason for the association-if indeed it exists-it is hard to overlook the persistent loss of fluid and electrolyte in the diarrhoea or ileostomy loss of these patients. The differences in incidence, however, suggest that a variety of other factors such as geography, diet, and climate might also be involved. After all, these factors are readily accepted as being important in determining the 
incidence and type of primary urinary calculi occurring in different parts of the world. It is likely that a combination of factors is responsible, possibly including biochemical changes in the blood and urine resulting from the fluid and electrolyte losses. Dietary changes and perhaps some other metabolic disturbances also need to be considered. These possibilities will not be elaborated on here, but the biochemical changes were discussed briefly in our previous communication (Bennett and Jepson, 1966) and by Clarke and McKenzie (1969). They are also the subject of further investigations which are currently proceeding.

We are most grateful to the many members of the Ileostomy Association of Victoria who kindly replied to circulars, and to those members and their doctors who willingly supplied additional information when requested.

\section{References}

Atsmon, A., De Vries, A., and Frank, M. (1963). Uric Acid Lithiasis. Amsterdam, Elsevier.

Badenoch, A. W. (1960). British fournal of Urology, 32, 374. Bennett, R. C., and Jepson, R. P. (1966). Australian and New Zealand

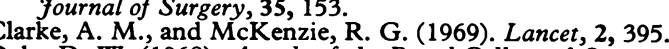

Clarke, A. M., and McKenzie, R. G. (1969). Lancet, 2, 395. 38.

Deren, J. J., Porush, J. G., Levitt, M. F., and Khilnani, M. T. (1962). Annals of Internal Medicine, 56, 843.

Edwards, F. C., and Truelove, S. C. (1964). Gut, 5, 1. Gelzayd, E. A., Breuer, R. I., and Kirsner, J. B. (1968). American fournal of
Digestive Diseases, 13, 1027 . Goligher, J. C., de Dombal, F. T., Watts, J. M., and Watkinson, G. (1968).
Ulcerative Colitis. London, Baillière, Tindall, and Cassell.

Lavan, J. N., Neale, F. C., and Posen, S. (1971). Medical Fournal of Australia, 2, 1049 .

Maratka, Z., and Nedbal, J. (1964). Gut, 5, 3.

Proudman, W. D. (1964). Communication to 3rd Scientific Meeting of Surgical Research Society of Australasia, Adelaide, 1964

Surgical Research Society of Austral
Ritchie, Jean K. (1971). Gut, 12, 536.

Williams, R. E. (1966). Quoted by Goligher et al. (1968).

\title{
Lithium-induced Hyperpolarization of the Human Rectum in Vivo
}

\author{
J. RASK-MADSEN， P. C. BAASTRUP， M. SCHWARTZ
}

British Medical fournal, 1972, 2, 496-498

\section{Summary}

The transmucosal potential difference across the rectal mucosa was measured in 30 healthy subjects and in 13 psychiatric patients on lithium treatment for manicdepressive psychosis. It was significantly greater in the lithium-treated patients. A highly significant correlation was found between the potential difference and the serum lithium, and in all eight patients in whom it was measured before and one week after starting lithium treatment a rising potential difference was found. This phenomenon may possibly be explained in terms of resistance of the rectal mucosa to vasopressin.

\section{Introduction}

As the effect of lithium salts on permeability characteristics and bioelectrical properties of biological membranes has been studied mainly in vitro, by using isolated animal tissues (Zerahn, 1955; Clarkson and Rothstein, 1960; Lindley and Hoshiko, 1964; Leb et al., 1965; Hayashi et al., 1971) or cells (Keynes and Swan, 1959; Carmeliet, 1964; Gardner and Kerkut, 1968) exposed to high concentrations of this ion, it seemed of interest to investigate how lithium treatment of psychiatric patients interferes with the potential difference generated by an intact membrane in the human organism. The rectum is a satisfactory organ for such a study as its epithelium is easily available, well defined, and generates great potential differences across the rectal wall, mucosa being negative to serosa (Geall et al., 1969; Dalmark, 1970; Edmonds and Godfrey, 1970). The action of a powerful sodium-absorbing pump seems to be the source of this transmucosal potential difference (Cooperstein and Hogben, 1959; Curran and Schwartz, 1960; Grady et al., 1970), which con-

Medical Department F, Glostrup Hospital, Glostrup, Denmark J. RASK-MADSEN, M.D., Research Fellow, University of Copenhagen M. SCHWARTZ, M.D., Professor of Medicine

Department O, Glostrup Psychiatric Hospital, Glostrup, Denmark P. C. BAASTRUP, M.D., Consultant Psychiatrist sequently reflects the major function of the rectal mucosanamely, the establishment of a steep concentration gradient for sodium between lumen and blood preventing excessive loss of electrolytes in the stools.

The spontaneous transmucosal potential difference across the rectal mucosa in patients with manic-depressive psychosis might differ from that of normals. In the present study therefore, we used the same technique on 30 healthy subjects and 13 manic and depressed patients; eight of the psychiatric patients were examined before and during lithium medication.

\section{Patients and Methods}

Seven patients were hypomanic and five moderately depressed at the time of starting lithium therapy. All were ambulatory and in a good general condition, and so were the normal subjects, who comprised healthy volunteers and patients in a medical ward without any recognized disease of the large bowel, liver, kidneys, or circulatory system. The psychiatric patients included in the study received no drugs other than lithium carbonate (16-65 mEq daily) during or within one week of beginning the study. The transmucosal potential difference was measured in five patients who had been on lithium carbonate continuously for one to six years, while it was determined in the other eight patients 12 hours before the first lithium dose and again seven to eight days later. Blood samples were obtained for serum lithium determination about one hour before the investigation, and analysed by flame photometry. Details of the different procedures of the electrical measurement and experiences of reproducibility will be published elsewhere (Rask-Madsen and Dalmark, 1972).

The transmucosal potential difference was obtained at sigmoidoscopy to secure that the mucosa appeared normal and was not contaminated with intestinal contents. As the serosal surface is equipotential to blood, one of two balanced calomel half-cells was connected to the low impedance input of a high impedance electrometer ( $\mathrm{pH}-$ Meter 51, Radiometer, Copenhagen) and to a vein of the forearm via a flowing $154 \mathrm{mM} \mathrm{NaCl}$ salt bridge, which was inserted for security reasons. The other half-cell connected the high impedance input of the electrometer with the exploring salt bridge, which consisted of a 